\title{
Suppression of very low frequency radio noise in transient electromagnetic data with semi-tapered gates
}

\author{
Jakob Juul Larsen ${ }^{1,5}$, Stine Søgaard Pedersen ${ }^{2,3}$, Nikolaj Foged ${ }^{2,5}$, and Esben Auken ${ }^{4}$ \\ ${ }^{1}$ Department of Engineering, Aarhus University, Finlandsgade 22, 8200 Aarhus N, Denmark \\ ${ }^{2}$ Hydrogeophysics Group, Department of Geoscience, Aarhus University, C. F. Møllers Alle 4, 8000 Aarhus C, Denmark \\ ${ }^{3}$ Department of Physics and Astronomy, Aarhus University, Ny Munkegade 120, 8000 Aarhus C, Denmark \\ ${ }^{4}$ Geological Survey of Denmark and Greenland, Øster Voldgade 10, 1350 Copenhagen, Denmark \\ ${ }^{5}$ WATEC, Aarhus University Centre for Water Technology, Ny Munkegade 120, 8000 Aarhus C, Denmark
}

Correspondence: Jakob Juul Larsen (jj1@eng.au.dk)

Received: 8 December 2020 - Discussion started: 28 December 2020

Revised: 24 February 2021 - Accepted: 8 March 2021 - Published: 15 April 2021

\begin{abstract}
The transient electromagnetic method (TEM) is widely used for mapping subsurface resistivity structures, but data are inevitably contaminated by noise from various sources. It is common practice to gate signals from TEM systems to reduce the amount of data and improve the signalto-noise ratio (SNR). Gating acts as a filter, and optimum gating will pass the TEM signal un-attenuated while suppressing noise. In systems based on analog boxcar integrators, the gating corresponds to filtering with a square window. The frequency response of this window shape has large side lobes, which are often insufficient in attenuating noise, e.g., from radio signals in the very low frequency (VLF) 3$30 \mathrm{kHz}$ band. Tapered gates have better side lobe suppression and attenuate noise better, but tapering with analog boxcar integrators is difficult. We propose using many short boxcar gates, denoted sub-gates, and combine the sub-gates into semi-tapered gates to improve noise rejection at late gates where low signal normally leads to poor SNR. The semitapering approach is analyzed and tested experimentally on data from a roving TEM system. We quantify the effect of semi-tapered gates by computing an improvement factor as the ratio between the standard error of data measured with boxcar gates and the standard error of data measured with semi-tapered gates. Data from a test survey in Gedved, Denmark, with 1825 measurements gave mean improvement factors between 1.04 and 2.22 for the 10 late-time gates centered between 78.7 and $978.1 \mu$ s. After inversion of the data, we find that semi-tapering increases the depth of investigation by about $20 \%$ for this specific survey. We conclude that
\end{abstract}

the semi-tapered approach is a viable path towards increasing SNR in TEM systems based on analog boxcar integrators.

\section{Introduction}

The transient electromagnetic method (TEM) is a widely applied geophysical method for delineating resistivity and resistivity structures in the subsurface of the earth. The method has found extensive use in many areas including mineral exploration, groundwater mapping, and geotechnical surveys; see Auken et al. (2017) for a recent review. Importantly, TEM instruments can be mounted on roving systems, which allows for high-resolution and cost-efficient mapping of large areas. Roving TEM systems include both fixed wing and helicopter airborne systems, as well as ground-based systems (e.g., Balch et al., 2003; Mulé et al., 2012; Auken et al., 2019).

The principle behind TEM is that a current applied to a transmitter coil generates a primary magnetic field. When the primary field is turned off, eddy currents are generated in the earth, which in turn generate a secondary magnetic field. The decay of the secondary field is measured by a receiver, typically an induction coil. The resistivity structure of the sub-surface earth is encoded in the secondary field and can be retrieved through inversion (Nabighian and Macnae, 1991).

Like any other electric or electromagnetic geophysical method, TEM is affected by electromagnetic noise. The noise is comprised of contributions from multiple sources includ- 
ing sinusoidal power grid components at 50 or $60 \mathrm{~Hz}$ and harmonics thereof, spherics from thunderstorms, very low frequency (VLF, 3-30 kHz) radio communication signals, internal noise from electronic components, and motion noise in roving systems (Macnae et al., 1984; Rasmussen et al., 2018a). Signal processing methods have been developed to mitigate the effects of noise. Important examples include suppression of power grid noise through synchronous detection using alternating polarity transmitter pulses, culling of signals affected by spherics, modeling and subtraction of VLF noise, and gating and stacking of signals (see for example Macnae et al., 1984; Nyboe and Sørensen, 2012; Macnae, 2015; Rasmussen et al., 2018b).

TEM measurements cover a huge dynamic range; signal values can span over 6 orders of magnitude on a timescale ranging from microseconds to tens of milliseconds. Historically, constraints in available technology implied that it was not possible to continuously sample the decaying TEM signal at the required dynamic range and store these data sets for later use. Instead, a common strategy was to use an analog integrator to boxcar gate the signal in exponentially increasing gate widths. The TEM signal approximately decays as $t^{-5 / 2}$. The analog integration over time gives an output decaying as $t^{-3 / 2}$, reducing the need for dynamic range in the subsequent analog-to-digital (A/D) conversion. In addition, the sampling period of the A/D converter can be reduced to that of the narrowest gate. In typical applications, 8-10 boxcar gates per time decade are used, enough to provide adequate representation of the TEM signal for inversion yet offering a significant reduction in the amount of data to be stored (Munkholm and Auken, 1996).

The boxcar gating corresponds to filtering of the data with a square window (Harris, 1978). The width of the boxcar gates in the time domain is inversely proportional to the width of the main lobe in the frequency domain. The exponentially increasing gate widths therefore correspond to filters with decreasing main lobe widths. This matches TEM signals, which extends to high frequencies at early times but has low-frequency content only at later times. Unfortunately, the frequency response associated with square windows has large side lobes, implying that noise is not necessarily efficiently suppressed. One particular example of this is VLF noise where different radio transmitters give rise to distinct noise peaks in the frequency spectrum. We note that while only a few papers have dealt with the suppression of VLF noise in TEM measurements, there are vast research fields and associated literature devoted to understanding the properties of VLF signals as well as utilizing the VLF signals in, for example, ionospheric studies and geophysical surveying (see for example Barr et al., 2000; Oskooi and Pedersen, 2005; Inan et al., 2010; Eppelbaum and Mishne, 2011).

The purpose of this work is to investigate the properties of an intermediate gating strategy, applicable to TEM systems equipped with analog boxcar integrators. Specifically, we increase the number of boxcar gates per decade and form weighted combinations of these to produce semi-tapered gates with improved noise suppression properties. The reduction of noise, in particular VLF noise in the $3-30 \mathrm{kHz}$ range, improves the data quality and extends the range of usable gates, which in turn leads to an increased depth of investigation.

The outline of the paper is as follows. First, we give a theoretical analysis and comparison of boxcar, tapered, and semitapered gates. Second, we present and compare experimental results of TEM data acquired with boxcar and semi-tapered gates.

\section{Methods}

\subsection{Gating}

At early times, the TEM signal extends from DC to high frequencies and has high amplitude. At late times, the TEM signal only extends from DC to low frequencies and has low amplitude. This is illustrated in the double-logarithmic plot in Fig. 1a where the TEM signal appears as a line. To improve on the signal-to-noise ratio and reduce the amount of data to be stored, it is customary to integrate the signal in adjacent time windows, called gates. The width of these gates must be chosen so the TEM signal is nearly constant within the gate. This requires short gates at early times where the signal decays fast. At late times, the signal decays slowly and longer gates can be used. Further, at late times the signal amplitude is reduced, and longer gates are beneficial as they integrate the signal over a longer time span and hence improve the SNR. These considerations lead to the common choice of exponentially increasing gate widths.

In systems with a single analog integrator, the gates are necessarily boxcar shaped and non-overlapping (Fig. 1b). For systems where the TEM signal is continuously digitized at a high sampling rate, any time window, i.e., any gate shape, can easily be applied in subsequent processing of data, and gates can overlap (Fig. 1c; Nyboe and Mai, 2017). Figure 1d presents an intermediate approach where an analog integrator outputs many short boxcar gates, denoted as sub-gates in the following. By forming a weighted combination of the sub-gates in subsequent processing, we create semi-tapered gates, with potentially improved noise suppression capabilities similar to continuously digitized sampling.

In essence, gating, i.e., windowing and integration of data, acts as a filter with a frequency response given by the Fourier transform of the gate shape (Harris, 1978). Hence, by applying carefully designed window shapes, selected frequency bands can be suppressed. We emphasize the following general properties about windows and their magnitude frequency response: 
(a)

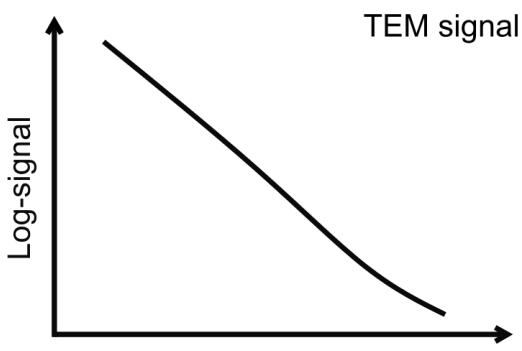

(b)

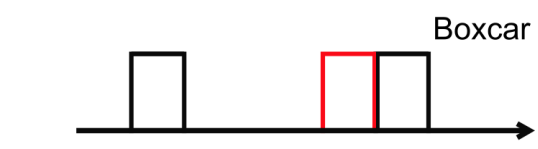

(c)

(d)

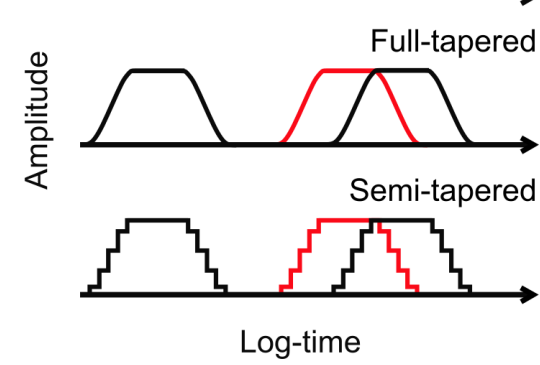

Figure 1. The plot in (a) illustrates a decaying TEM signal. The plots in (b)-(d) show the rectangular, full-tapered, and semi-tapered gates, respectively. For simplicity only three gates are shown for each gating strategy, and they are colored to make them distinguishable. Both full-tapered and semi-tapered gates overlap with neighboring gates. The gate widths are exponentially increasing; hence gates appear with identical widths on the logarithmic time axis.

- Increasing the length of a window will decrease the width of the main lobe in the frequency domain.

- In the case of windows of equal length, the boxcar window has the narrowest main lobe, but also the largest side lobes.

- Tapered windows have lower-amplitude side lobes than boxcar windows.

Figure 2 illustrates this in detail for a single gate centered at $419.7 \mu$ s. In Fig. 2a three different gate shapes are plotted: a boxcar gate (full blue line), a full-tapered gate (dashed black line) consisting of a flat main segment identical to the boxcar gate, a smooth half-cosine tapering at both edges, and a semi-tapered gate (full red line) also consisting of a flat main segment identical to the boxcar gates and with nonsmooth tapering at both edges. Each taper is made up of five sub-gates with values matching the smooth half-cosine at the sub-gate centers. The half-cosine tapers are symmetric in logarithmic time, as shown in the figure, and are therefore non-symmetric in linear time. Corresponding plots of the magnitude frequency response are shown in Fig. 2b. The responses have been normalized for unit gain at $0 \mathrm{~Hz}$. We note the following two specific features from the magnitude frequency response plot: first, the width of the main lobe
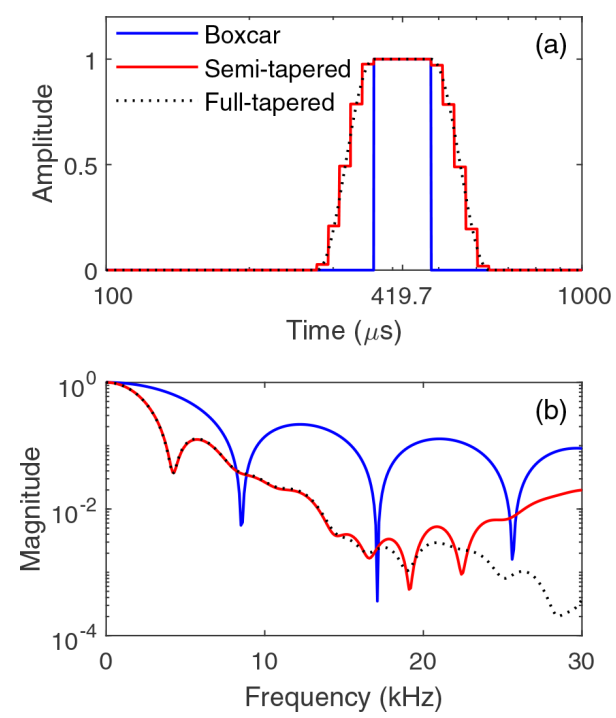

Figure 2. Comparison of boxcar, full-tapered, and semi-tapered gates in the time domain (a) and frequency domain (b). The gate center time is $419.7 \mu \mathrm{s}$. The boxcar gate width is $114.6 \mu \mathrm{s}$ and the semi-tapered width is $235.5 \mu$ s (gate 17 in Table 1).

is wider for the boxcar gate than for the tapered and semitapered gate. This counter-intuitive result is caused by the increased widths of the tapered gates, narrowing the main lobe. For the three gates shown in Fig. 2, the widths, measured as full width at half maximum, are $114.6 \mu$ s (boxcar) and $235.5 \mu$ s (full- and semi-tapered). Second, both the fulltapered and semi-tapered windows have, on average, almost identical and significantly lower amplitude side lobes, which improves overall noise suppression in the VLF band.

The half-cosine tapers (full or semi-) added to the center boxcar gate are only one particular choice of gate shape. We also performed simulations and experiments with other gate shapes, including Gaussian gates and boxcar gates augmented with linear tapers (Harris, 1978). We found that the performance of boxcar gates augmented with half-cosine tapers gave good results for the tested scenarios, but also that other gate shapes could have better performance in specific scenarios. This is not a surprising result as the effectiveness in noise suppression depends on the exact match between the spectrum of the noise and the frequency response of the gate. For instance, if the frequency of a given radio transmitter coincides with one of the dips in the gate magnitude frequency response, the transmitter will be efficiently suppressed.

For simplicity and visual clarity, the above comparison of gate shapes is based on analysis of single gates. In practice, TEM systems use a train of alternating TEM transmit pulses and apply repetitive sampling and sign correction of the received TEM signal for suppression of power-line noise (Macnae et al., 1984). In the frequency domain, the repetitive sampling causes a comb-like spectrum (Appendix A). Importantly, the effects of repetitive sampling are identical, 
regardless of the specific gate shape. The above conclusions of the noise suppression of different gate shapes therefore remain valid also after repetitive sampling.

\subsection{The tTEM system}

Measurements are conducted with a ground-based towed transient electromagnetic (tTEM) system fully described in Auken et al. (2019). Briefly, the tTEM system is towed behind an all-terrain vehicle and comprises a $2 \mathrm{~m}$ by $4 \mathrm{~m}$ transmitter coil and a $0.5 \mathrm{~m}$ by $0.5 \mathrm{~m}$ receiver coil placed $7 \mathrm{~m}$ behind the transmitter coil. The system uses dual transmitter moments (low and high) to obtain early- and late-time TEM data, roughly corresponding to shallow and deep geological layers. In the low moment, the transmitter pulse repetition rate is $2110 \mathrm{~Hz}$ and the first usable gate lies at $\approx 4 \mu$ after the beginning of current turn-off. In the high moment, the transmitter pulse repetition rate is $660 \mathrm{~Hz}$, and the first usable gate lies at approximately $9 \mu$ s after current turn-off. Highmoment measurements last up to $1000 \mu$ s after turn-off with the limit set by the pulse repetition rate. The receiver system contains an analog integrator to boxcar gate the TEM signal. In the standard configuration, a low-moment measurement contains 15 boxcar gates with center times from 4 to $33 \mu$ s, and a high-moment measurement contains 23 boxcar gates with center times from 10 to $900 \mu \mathrm{s}$. The output from the analog integrator is sampled with an ADC and stored. The tTEM system uses synchronous detection with alternating polarity transmitter pulses, and the recorded gate values are therefore sign-corrected before stacking (Appendix B). A low-moment stack, i.e., one sounding, is made up of 422 individual transients, and one high-moment stack is made up of 252 individual transients. In normal operation, the system interleaves between low-moment and high-moment stacks.

The tTEM system can be configured to output up to a maximum of 84 boxcar gates distributed in the low and high moment. The start time and gate width of each boxcar gate is individually controlled with a $0.65 \mu$ s minimum gate width and a $0.35 \mu$ s boxcar integrator reset time between gates. The limit of 84 gates is imposed by the communication speed of the electronics.

\section{Experimental results}

A test survey was carried out on farming fields in Gedved, Denmark. The area is characterized by glacial sediment deposits, and the resistivity ranges from $8-500 \Omega \mathrm{m}$. The survey site was selected based on the condition that the later gates, i.e., from approximately $100 \mu$ s and onwards, should be dominated by noise, including VLF radio noise. This ensures that differences in noise rejection between different gating strategies are readily discernible. The driving conditions on the site were good and no motion-induced noise artifacts were observed in the processed data.

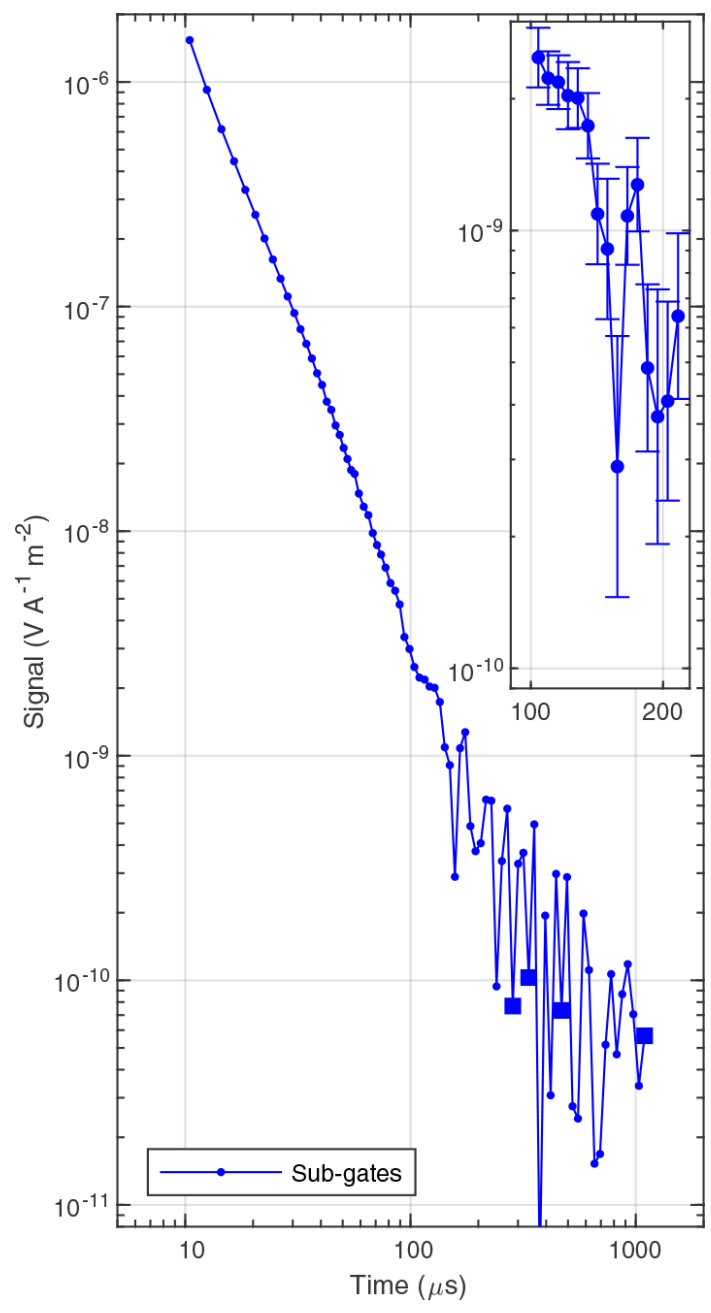

Figure 3. Sounding curve from the Gedved survey consisting of 81 boxcar-shaped sub-gates. For negatively valued sub-gates, the absolute value is plotted and indicated with a square marker. From approximately $100 \mu$ s and onwards the data are dominated by VLF noise and oscillates. The inset shows values and standard errors for sub-gates between 100 and $200 \mu$ s.

For this study, we focus on the high-moment data part, where VLF radio noise can be non-negligible at late gates. The tTEM system was configured to record 84 boxcar gates, denoted sub-gates in the following. The center times and widths exponentially increase within the limitations enforced by the receiver electronics. The first useable high-moment sub-gate is gate 4 centered at $9.2 \mu$ s with a $1.65 \mu$ s width. The last sub-gate is $67.0 \mu$ s long and centered at $1095.2 \mu$ s. For each stack, the 252 transients are sign-corrected and a motion-noise filter is applied (Auken et al., 2019). The filter output contains 204 transients from which we compute mean values and standard errors (Appendix B).

A representative high-moment sounding curve from the Gedved survey containing 81 sub-gates is plotted in Fig. 3. We observe a smooth decay in the TEM signal until $\sim 100 \mu$ s. 


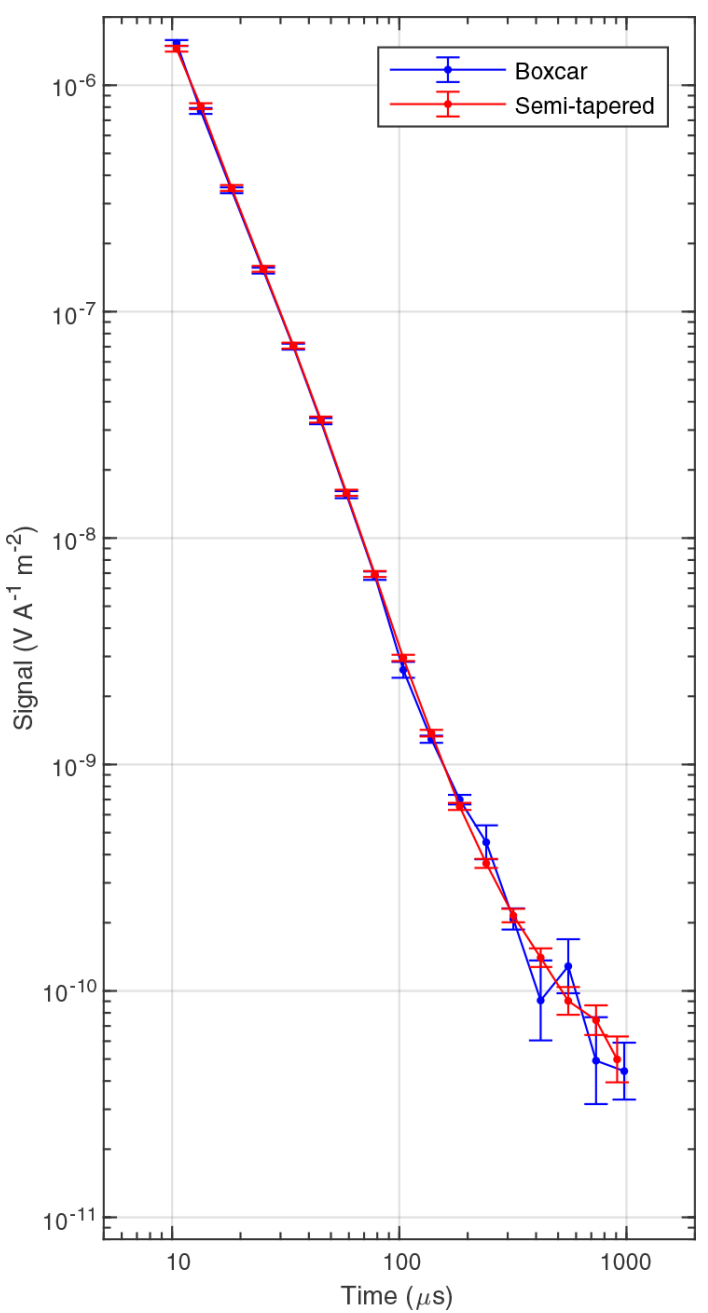

Figure 4. Sounding curves, boxcar and semi-tapered, from the Gedved survey. Each sounding curve contains 17 gates. The boxcar and semi-tapered gates are constructed from the sub-gates shown in Fig. 3.

From $100 \mu$ s and onwards oscillations are seen in the sounding curve. The inset in the figure shows the TEM signal from 100 to $200 \mu$ s and the associated standard errors. Importantly, the errors are significantly smaller than the observed oscillations in data and hence the oscillations cannot be interpreted as the result of random noise. We ascribe the oscillations to VLF noise, which in this context can be considered a coherent noise source, which is not efficiently suppressed by stacking of transients. Our interpretation is supported by an analysis of the peak-to-peak separations of the oscillations. The separations correspond to frequencies in the $20-25 \mathrm{kHz}$ range in agreement with VLF radio stations observed in Denmark (Rasmussen et al., 2018b).

Next, for each transient, we assemble linear combinations of the short sub-gates into longer, exponentially widening gates (either boxcar or semi-tapered) with a target of 10 gates per decade. In the case of boxcar-shaped gates, the gates from $100 \mu$ s and onwards are composed of five or six subgates controlled by the exact distribution of sub-gates. For semi-tapered gates, the flat top contains the same five or six sub-gates and the tapers overlap with the previous and next boxcar gate. Each taper contains five or six sub-gates with values matching a smooth half-cosine taper at the sub-gate centers. The only exceptions are the last two gates, where the finite number of available sub-gates enforces a non-cosine off-tapering. For the last gate, only three sub-gates are available for the boxcar gate and the flat-top part of the semitapered gate. The subsequent processing of assembled data is similar to above. Data are sign-corrected, motion-noise filtered, and stacked.

In Fig. 4 we plot an example of a boxcar and a semitapered sounding curve, with gate 4-20 in each. The first three gates are distorted by transmitter turn-off effects and therefore culled. The two sounding curves are very nearly identical until $\sim 200 \mu \mathrm{s}$. After this time, the semi-tapered curve is continuous with a smooth decay, whereas the boxcar sounding curve still shows oscillations. Further, the error bars on the boxcar gate values are larger than for the semi-tapered gates. Compared to the 81-gate sounding curve in Fig. 3, we see that the increased width of the boxcar gates extends the range of usable data but is incapable of sufficient reduction after $\sim 200 \mu$ s. In contrast, the semi-tapering is significantly better at suppressing VLF noise. The sounding curve is much smoother and follows the normal decay of a transient.

Figure 5 shows 200 subsequent soundings from the Gedved survey with sub-gates gated into either standardlength boxcar gates (Fig. 5a) or semi-tapered gates (Fig. 5b). Gates 10-17 with gate centers from 59.2 to $978.1 \mu$ s and gate widths from 14.6 to $394.9 \mu$ s are plotted (Table 1). It takes a little less than 2 min to record the 200 soundings, during which the tTEM system moves approximately $200 \mathrm{~m}$. The plots show a clear suppression of noise with the semi-tapered gating strategy as compared to the boxcar gating strategy for all gates. Earlier gates are visibly very similar for the two gating strategies and therefore not plotted. Gates 18-20 also show improvement, but these gates are not shown, as the noise is too large in these late gates for an easy visual comparison on the same plot. The noise is less pronounced in the first $\sim 75$ soundings, indicating a strong nonstationary noise field.

We quantify the improvement in noise reduction by defining an improvement factor, $\gamma$, as the standard error of data measured with boxcar gates divided by the standard error of data measured with semi-tapered gates, i.e.,

$\gamma=\frac{S_{\text {boxcar }}}{S_{\text {semi-tapered }}}$.

The improvement factor is above one if the standard error of data measured with semi-tapered gates is less than the standard error of data measured with rectangular gates, i.e., if the noise reduction is improved with semi-tapered gates and vice versa. In Fig. 6 we plot histograms of the improve- 

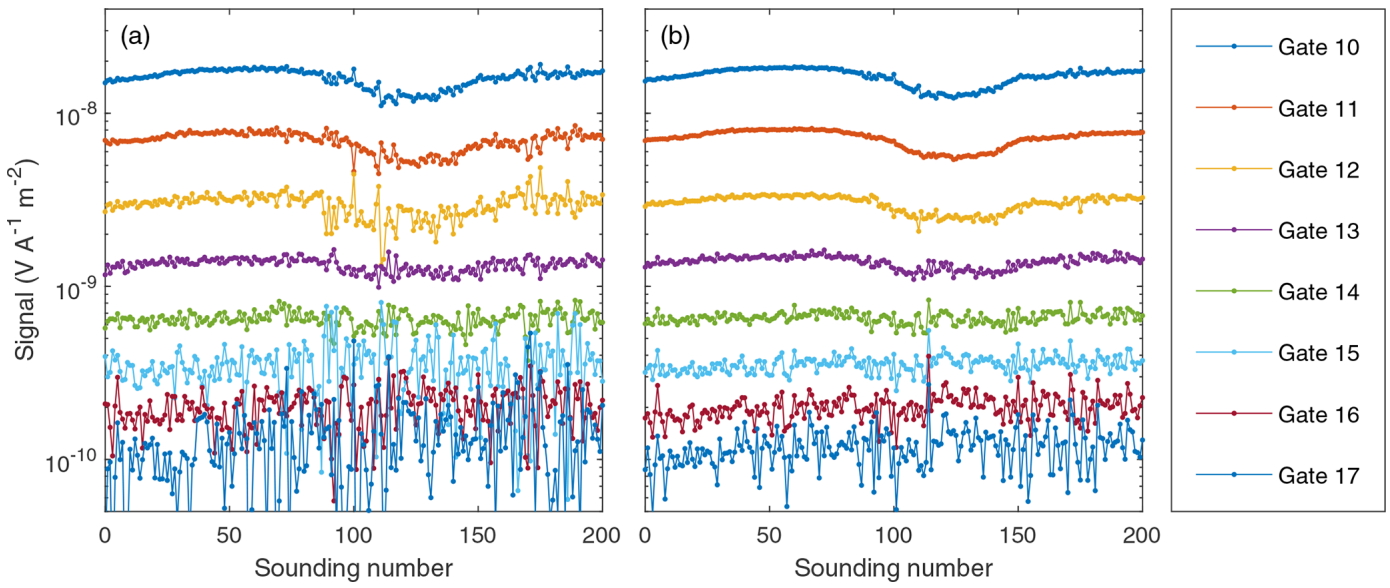

Figure 5. Gate values as a function of sounding index acquired with the tTEM system moving (a) boxcar gates and (b) semi-tapered gates; see Table 1 for gate details. The 200 soundings correspond to approximately $200 \mathrm{~m}$ of driving. The noise using semi-tapered gates is visibly lower than for boxcar gates.

ment factors for gate 9-20 measured on 1825 soundings from the Gedved survey. On top of the histograms, mean value and standard error of the improvement are indicated with red lines. Starting from gate 15 , centered at $242 \mu$ s, we see significant improvement factors, with the mean close to or exceeding two in four gates. In gate 16, the mean improvement is 1.31 , which is remarkably low compared to the improvement in neighboring gates. This is likely caused by a specific high-amplitude VLF radio transmitter being well suppressed by a zero in the boxcar frequency response. For the last gate, 20, we also see a low improvement factor of 1.19. This low value is due to the truncated semi-tapered gate having a nonoptimum frequency response close to the boxcar frequency response. The results are summarized in Table 1. We observed no improvement for the early gates (before gate 9). This result is anticipated as the main lobe using either gating strategy exceeds well beyond the $3-30 \mathrm{kHz}$ VLF band.

If the improvement factors are analyzed as functions of the gate values, on average, negative correlations are seen. This result is intuitively clear, as high gate values generally correspond to large TEM signals where the impact from VLF noise is smaller and hence the potential improvement is smaller.

We assess the impact of the semi-tapering in the resistivity model space by smooth inversion of the two differently gated data sets. During processing of data, all gates with a standard error exceeding $10 \%$ of the gate value are culled together with any later gates in the sounding. Similarly, all negative data values are culled. As part of the inversion process we also compute the depth of investigation (DOI) for each sounding with the approach developed by Christiansen and Auken (2012). In Fig. 7, we present a scatter plot of DOIs for the 1825 soundings. On average, in this case we find that semi-tapered gating increases the DOI by $15 \mathrm{~m}$ or about $20 \%$.
Table 1. Gate center times, gate width, improvement factors, $\gamma$, and standard deviation for gates 9 to 20 . Gate widths are measured as full width at half maximum. Boxcar and semi-tapered gates have the same center time except for the last gate (20), where the table gives the center time of the boxcar gate. The corresponding semitapered gate center time is $910.4 \mu \mathrm{s}$.

\begin{tabular}{rrrrr}
\hline $\begin{array}{r}\text { Gate } \\
\text { no. }\end{array}$ & $\begin{array}{r}\text { Gate center } \\
(\mu \mathrm{s})\end{array}$ & $\begin{array}{r}\text { Boxcar } \\
\text { gate width } \\
(\mu \mathrm{s})\end{array}$ & $\begin{array}{r}\text { Semi-tapered } \\
\text { gate width } \\
(\mu \mathrm{s})\end{array}$ & $\begin{array}{r}\text { Impr. } \\
\text { factor } \\
\gamma\end{array}$ \\
\hline 9 & & 11.6 & 23.4 & $0.99 \pm 0.02$ \\
10 & 45.8 & 14.6 & 29.5 & $1.02 \pm 0.05$ \\
11 & 59.2 & 24.6 & 49.8 & $1.07 \pm 0.12$ \\
12 & 104.7 & 26.6 & 53.7 & $1.26 \pm 0.32$ \\
13 & 139.1 & 42.6 & 86.2 & $1.04 \pm 0.08$ \\
14 & 185.1 & 48.6 & 98.1 & $1.12 \pm 0.12$ \\
15 & 242.0 & 65.6 & 132.4 & $2.08 \pm 1.01$ \\
16 & 318.2 & 87.6 & 176.9 & $1.31 \pm 0.27$ \\
17 & 419.7 & 114.6 & 235.5 & $2.22 \pm 1.06$ \\
18 & 554.8 & 155.6 & 314.3 & $1.88 \pm 0.68$ \\
19 & 735.3 & 208.6 & 421.4 & $1.98 \pm 0.88$ \\
20 & 978.1 & 281.6 & 394.9 & $1.18 \pm 0.16$ \\
\hline
\end{tabular}

We also compute the average logarithmic data residual, $\phi$, of the inverted models using

$\phi=\sqrt{\frac{1}{N_{\mathrm{g}}} \sum_{k=1}^{N_{\mathrm{g}}}\left(\frac{\ln \left(X_{k}^{\text {observed }}\right)-\ln \left(X_{k}^{\text {predicted }}\right)}{\ln \left(1+S_{k} / X_{k}^{\text {observed }}\right)}\right)^{2}}$,

where $N_{\mathrm{g}}$ is the number of gates and $X_{k}$ denotes the $k$ th observed or predicted gate value with standard error $S_{k}$ (Appendix B). For boxcar gating the average data residual is 1.38 , whereas this value is only 1.09 using semi-tapered gating. Our interpretation of this difference is that the standard errors on the boxcar gate values are not truly representative of 

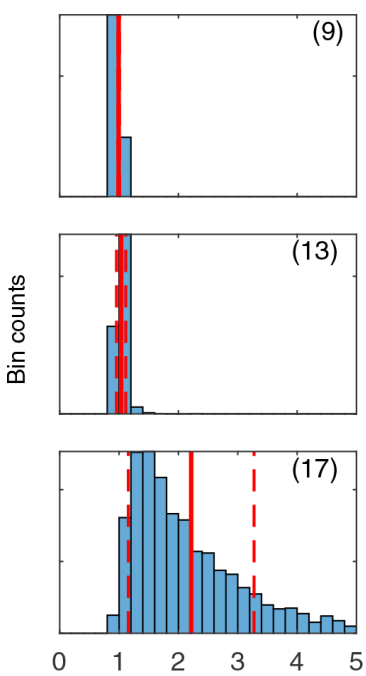
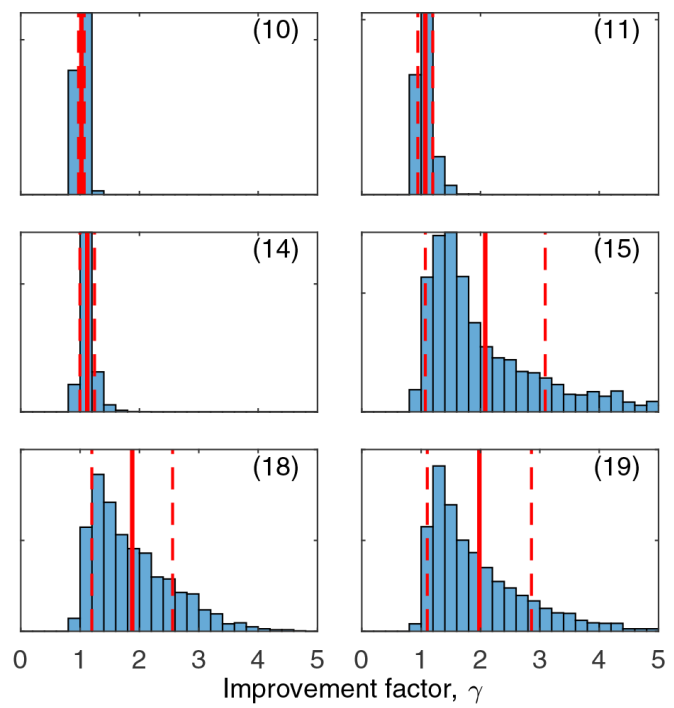
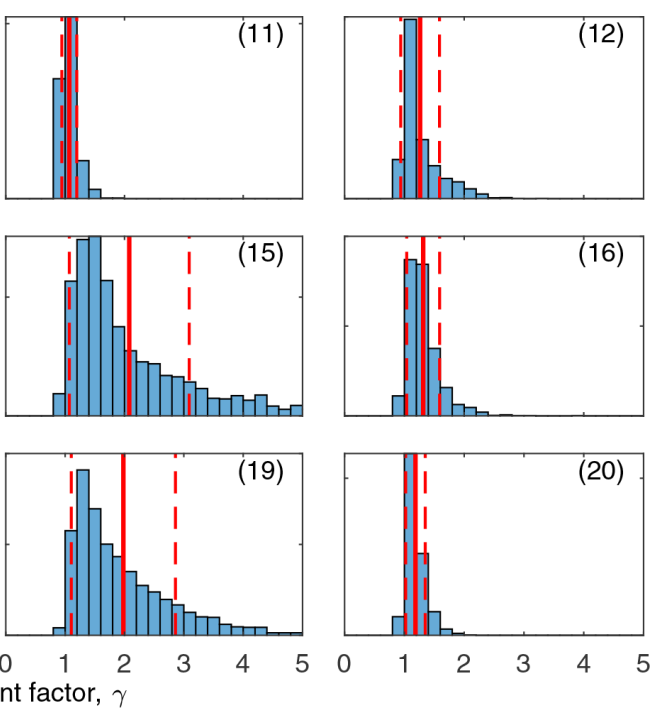

Figure 6. Histogram of improvement factors for gates 9-20 presented as bin counts. The bin count axis is individually scaled for each histogram to make the distribution visible. The full red line shows the mean value of improvement factor; the dashed red lines show the standard deviation.

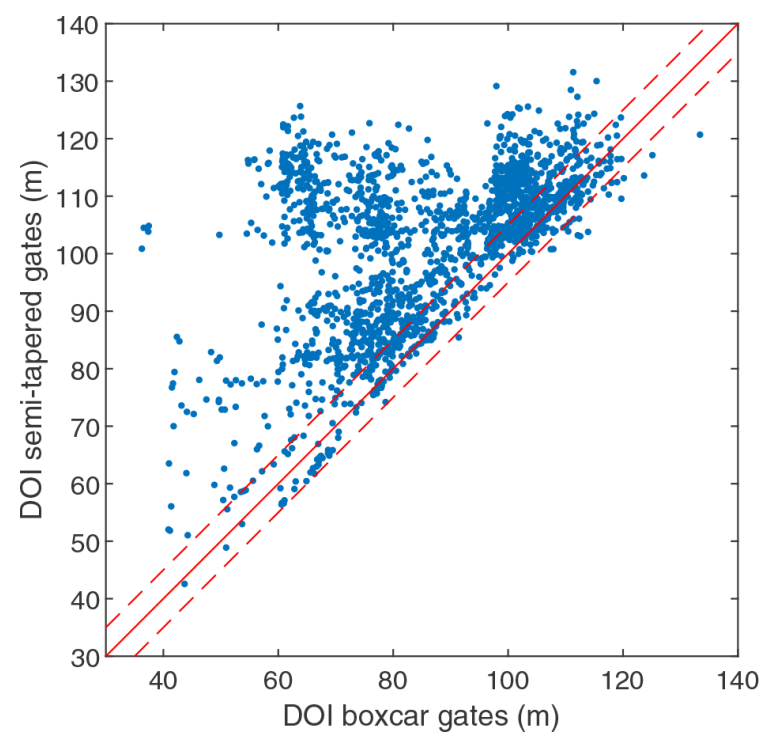

Figure 7. Scatter plot of depth of investigation (DOI) using either boxcar or semi-tapered gating. The full red line indicates equality of DOI, while the two red dashed lines indicate a DOI difference of $5 \mathrm{~m}$. The average increase in DOI using semi-tapered gating is $15 \mathrm{~m}$.

the noise. This occurs as the standard error reflects only the random noise in the data, but coherent VLF noise is not properly accounted for. Using semi-tapered gating, the coherent VLF noise is suppressed, and the standard error reflects the actual noise in the data much better.

\section{Discussion}

The semi-tapered gates that we employ are based on augmenting boxcar gates with tapering on both sides of the original boxcar gate. The improvement in noise suppression is therefore a combination of two properties. First, the augmenting with tapers increases the width of the semi-tapered gates as seen in Table 1. This leads to a narrower main lobe and hence by itself improved suppression of low-frequency noise. Second, the tapering gives a magnitude response with suppressed side lobes and hence also an improved noise rejection.

In the data from the Gedved survey, sub-gates are visibly influenced by VLF noise from around $100 \mu$ s and onwards, and our analysis has focused on this part of the data. It is also possible to use the semi-tapering approach with earlier gates. For this data set, the sub-gates recorded before $\sim 100 \mu$ s generally have high SNR, and the need for additional noise reduction is absent. In our approach where we use additional sub-gates for tapering, the semi-tapering increases the width of any given gate. In turn, this reduces the main lobe width, which can influence the desired TEM signal, especially at early times during which the TEM signal has high bandwidth. However, for surveys conducted on high-resistivity sub-surfaces, the TEM signal will disappear into noise at earlier times, and starting semi-tapering of sub-gates earlier than $100 \mu$ s can be beneficial.

In noise scenarios, where the majority of noise stems from a single powerful narrowband radio transmitter, it can still be beneficial to employ boxcar gates or to use more advanced semi-tapering schemes. Figure $2 \mathrm{~b}$ shows that boxcar gates have zeros in the magnitude response. Hence, by choosing the width of a boxcar gate appropriately, a zero can be placed 
directly at the frequency of the radio transmitter, resulting in a very high noise rejection. Likewise, more advanced gating strategies are also possible. By tuning the width of boxcar sub-gates as well as the width and tapering of combined gates, a set of semi-tapered gates can be optimized for the exact noise conditions at the site where a survey is carried out. As a simple example of the potential of this approach, we carried out an experiment on the Gedved data where a new gate 16 was assembled by shifting all sub-gates one step earlier. This shifted the gate center from 318.2 to $300.9 \mu \mathrm{s}$, while the gate width was reduced from 87.2 to $82.6 \mu$ s (boxcar) and from 179.9 to $166.8 \mu \mathrm{s}$ (semi-tapered). The outcome of the shifting operation is a significant increase in the improvement factor from $1.31 \pm 0.27$ to $1.63 \pm 0.60$ for gate 16 . For the other gates, some small increases as well as some small decreases in the improvement factor were observed.

For a given survey, the actual improvement in SNR and in turn improved depth of investigation can vary significantly depending on the resistivity structures and the local noise environment. In particular, we have observed that rough driving conditions can induce motion noise so that post-filtering still exceeds the contributions from VLF noise. In such a case where TEM measurements are dominated by motioninduced noise, the performance of semi-tapered and boxcar gating strategies are on par. The data set that has been used for this work stems from an area with low to medium sub-surface resistivity, which gives rise to large-amplitude TEM signals. In surveys performed over high-resistivity subsurfaces, the TEM signal, particularly in the late gates, will be significantly lower and even better results than presented here can be expected in many cases.

One attractive feature of semi-tapering is its ability to suppress coherent VLF noise, which cannot be achieved by stacking of data. The VLF noise suppression leads to estimates of error bars that are more truly representative of the actual noise in data. Further, the suppression of VLF noise reduces systematic errors in data, as can be seen in Fig. 4 where the semi-tapered gating results in a smoother decay than the boxcar gating. In turn, this leads to resistivity models with better determined resistivity parameters and larger depth of investigation.
This study has shown that boxcar gates can efficiently be turned into semi-tapered gates with close to the same noise suppression capability as fully sampled transients. From an instrument design perspective this is quite important because modern systems can use A/D converters sampling at $10 \mathrm{MHz}$ or more. Storing this amount of data is not possible. Decimating the data stream into boxcar gates can reduce the storage to maybe 100-200 gates per transient, which is unproblematic and it allows for re-gating during data processing.

\section{Conclusions}

We have explored a new gating strategy applicable for TEM systems equipped with analog boxcar integrators. The gating strategy is based on acquiring many short boxcar gates, which are then combined into a set of overlapping semitapered gates. The semi-tapered gates were experimentally shown to have better noise suppression than the standard boxcar gates. The noise suppression was quantified by comparing the standard error of data acquired with each approach and through the depth of investigation. We found that for this specific data set, the standard error was decreased by a factor between 1.04 and 2.21 and the depth of investigation was increased by $20 \%$ using the semi-tapered gates. The increase in SNR is achieved at no expense, except a reprogramming of the gating scheme.

The reduced standard error in the data translates directly to an increased SNR. If the same improvement in SNR were to be obtained by increasing the signal through an increased transmitter current, the current would have to be increased by the same factor (in this case a factor of 2), which is a significant engineering challenge. 


\section{Appendix A: Frequency domain effects of repetitive sampling}

In concordance with the TEM transmitter signal consisting of $N$ alternating polarity pulses, we consider a sequence of $N$ identical gates with arbitrary shape $h(t)$ and alternating polarity. The interval between gates is denoted by $\tau$.

$$
\begin{aligned}
h_{N}(t)= & \frac{1}{N}(h(t)-h(t-\tau)+h(t-2 \tau)-\ldots \\
& +h(t-[N-2] \tau)-h(t-[N-1] \tau))
\end{aligned}
$$

The Fourier transform of $h_{N}(t)$, denoted by $\mathcal{F}\left\{h_{N}(t)\right\}=$ $H_{N}(j \Omega)$, with $\Omega=2 \pi F$ can be calculated using the linearity and time shifting properties of the Fourier transform (Manolakis and Ingle, 2011), i.e.,

$$
\begin{aligned}
H_{N}(j \Omega)= & \mathcal{F}\left\{\frac{1}{N}(h(t)-h(t-\tau)+\ldots\right. \\
& +h(t-[N-2] \tau)-h(t-[N-1] \tau))\} \\
= & \frac{1}{N}\left(H(j \Omega)-e^{j \tau \Omega} H(j \Omega)+\ldots\right. \\
& \left.-e^{j(N-2) \tau \Omega} H(j \Omega)-e^{j(N-1) \tau \Omega} H(j \Omega)\right) \\
= & \frac{1}{N}\left(1-e^{j \tau \Omega}+\ldots\right. \\
& \left.+e^{j(N-2) \tau \Omega}-e^{j(N-1) \tau \Omega}\right) H(j \Omega) .
\end{aligned}
$$

Using $e^{j \pi n}=1$ for even $n$ and $e^{j \pi n}=-1$ for odd $n$, the exponential terms in the parenthesis are collected as

$$
\begin{aligned}
H_{N}(j \Omega) & =\left(\frac{1}{N} \sum_{n=0}^{N-1} e^{j(\pi-\tau \Omega) n}\right) H(j \Omega) \\
& =\left(\frac{1}{N} \frac{1-e^{j(\pi-\tau \Omega) N}}{1-e^{j(\pi-\tau \Omega)}}\right) H(j \Omega) \\
& =\left(e^{j \alpha \frac{N-1}{2}} \frac{1}{N} \frac{\sin (\alpha N / 2)}{\sin (\alpha / 2)}\right) H(j \Omega),
\end{aligned}
$$

where $\alpha=\pi(1-2 \tau F)$ was introduced in the last step. The term inside the parenthesis can be recognized as the Dirichlet function, also known as the periodic sinc function, and an additional phase factor (Manolakis and Ingle, 2011). The result is that $H_{N}(j \Omega)$ is found by the product of $H(j \Omega)$ and the periodic Dirichlet function. The multiplication gives rise to a comb-like sampling of the spectrum of a single gate at frequencies, $F, 3 F, 5 F$, etc. In the context of this work, the important feature is that the effects of repetitive sampling, modeled by the Dirichlet function, are identical, irregardless of the shape of $H(j \Omega)$.

\section{Appendix B: Calculation of uncertainty on gate values}

The tTEM system repeats the measurement of each transient $N_{\mathrm{t}}$ times and each transient contains $N_{\mathrm{sg}}$ sub-gates. We denote the value of the $i$ th transient and $j$ th sub-gate as $\tilde{x}_{i j}$ with $i=1, \ldots, N_{\mathrm{t}}$ and $j=1, \ldots, N_{\mathrm{sg}}$. The measured data are sign-corrected, motion-noise filtered, and then processed as follows. Note that the motion-noise filter reduces $N_{\mathrm{t}}$ by the length of the employed filter as data with transient filter distortion are discarded. For each transient, the data are first regated into $N_{\mathrm{g}}$ longer boxcar or semi-tapered gates using

$x_{i k}=\sum_{j=1}^{N_{\mathrm{sg}}} \tilde{x}_{i j} w_{j k}$,

where $k=1, \ldots, N_{\mathrm{g}}$ and $w_{j k}$ is the weight of the $j$ th sub-gate in the $k$ th gate. The weighting factors are normalized to a unit magnitude response at $0 \mathrm{~Hz}$. Second, the mean (stacked) value of the $k$ th gate, $X_{k}$, is computed as

$X_{k}=\frac{1}{N_{\mathrm{t}}} \sum_{i=1}^{N_{\mathrm{t}}} x_{i k}$.

Next, the mean square deviation is computed with

$s_{k}^{2}=\frac{1}{N_{\mathrm{t}}-1} \sum_{i=1}^{N_{\mathrm{t}}}\left(x_{i k}-X_{k}\right)^{2}$.

Finally, the standard error for the stacked measurement is estimated as

$S_{k}=\frac{s_{k}}{\sqrt{N_{\mathrm{t}}}}$

The values of stacked data are reported along with their standard error as $X_{k} \pm S_{k}$ (Barford, 1990). 
Data availability. The raw data used in this paper are available upon request to the corresponding author.

Author contributions. The idea was conceived by EA and JJL. Data collection and data analysis were performed by SSP, NF, and JJL. Analysis and interpretation were done by SSP, NF, EA, and JJL. The paper was written by SSP and JJL.

Competing interests. The authors declare that they no conflict of interests.

Acknowledgements. Pradip K. Maurya is kindly acknowledged for help with tTEM field measurements and data processing. The tTEM data were collected during TOPSOIL, an Interreg project supported by the North Sea Programme of the European Regional Development Fund of the European Union.

Financial support. This research is supported by Innovation Fund Denmark through the MapField project.

Review statement. This paper was edited by Lev Eppelbaum and reviewed by James Macnae and Marco Antonio Couto Junior.

\section{References}

Auken, E., Boesen, T., and Christiansen, A. V.: A Review of Airborne Electromagnetic Methods With Focus on Geotechnical and Hydrological Applications From 2007 to 2017, Adv. Geophys., 58, 47-93, https://doi.org/10.1016/bs.agph.2017.10.002, 2017.

Auken, E., Foged, N., Larsen, J. J., Lassen, K. V. T., Maurya, P. K., Dath, S. M., and Eiskjær, T. T.: tTEM - a towed TEM-system for detailed 3D imaging of the top 70 meters of the subsurface, Geophysics, 84, E13-E22, https://doi.org/10.1190/GEO20180355.1, 2019.

Balch, S. J., Boyko, W. P., and Paterson, N. R.: The AeroTEM airborne electromagnetic system, The Leading Edge, 22, 562-566, https://doi.org/10.1190/1.1587679, 2003.

Barford, N. C.: Experimental Measurements: Precision, Error and Truth, 2nd edn., Wiley, Chichester, 1990.

Barr, R., Jones, L., and Rodger, C. J.: ELF and VLF radio waves, J. Atmos. Sol.-Terr. Phy., 62, 1689-1718, https://doi.org/10.1016/S1364-6826(00)00121-8, 2000.

Christiansen, A. V. and Auken, E.: A global measure for depth of investigation, Geophysics, 77, WB171-WB177, https://doi.org/10.1190/GEO2011-0393.1, 2012.

Eppelbaum, L. V. and Mishne, A. R.: Unmanned Airborne Magnetic and VLF investigations: Effective Geophysical Methodology of the Near Future, Positioning, 2011, 112-133, https://doi.org/10.4236/pos.2011.23012, 2011.
Harris, F. J.: On the use of windows for harmonic analysis with the discrete Fourier transform, Proc. IEEE, 66, 51-83, https://doi.org/10.1109/PROC.1978.10837, 1978.

Inan, U. S., Cummer, S. A., and Marshall, R. A.: A survey of ELF and VLF research on lightning-ionosphere interactions and causative discharges, J. Geophys. Res., 115, A00E36, https://doi.org/10.1029/2009JA014775, 2010.

Macnae, J.: Stripping very low frequency communication signals with minimum shift keying encoding from streamed timedomain electromagnetic data, Geophysics, 80, E343-E353, https://doi.org/10.1190/geo2015-0304.1, 2015.

Macnae, J. C., Lamontagne, Y., and West, G. F.: Noise processing techniques for time-domain EM systems, Geophysics, 49, 934 948, https://doi.org/10.1190/1.1441739, 1984.

Manolakis, D. G. and Ingle, V. K.: Applied digital signal processing, Cambridge University Press, New York, 2011.

Mulé, S., Miller, R., Carey, H., and Lockwood, R.: Review of three airborne EM systems, ASEG Extended Abstracts, 2012, 1-5, https://doi.org/10.1071/ASEG2012ab352, 2012.

Munkholm, M. and Auken, E.: Electromagnetic Noise Contamination on Transient Electromagnetic Soundings in Culturally Disturbed Environments, J. Environ. Eng. Geoph., 1, 119-127, https://doi.org/10.4133/JEEG1.2.119, 1996.

Nabighian, M. N. and Macnae, J. C.: Time Domain Electromagnetic Prospecting Methods, in: Electromagnetic Methods in Applied Geophysics: Volume 2, Application, Parts A and B, Society of Exploration Geophysicists, Tulsa, 427-520, https://doi.org/10.1190/1.9781560802686.ch6, 1991.

Nyboe, N. S. and Mai, S. S.: Recent Advances in SkyTEM Receiver System Technologies, in: Second European Airborne Electromagnetics Conference, European Association of Geoscientists and Engineers (EAGE), Malmö, 1-5, https://doi.org/10.3997/2214-4609.201702157, 2017.

Nyboe, N. S. and Sørensen, K.: Noise reduction in TEM- Presenting a bandwidth- and sensitivity-optimized parallel recording setup and methods for adaptive synchronous detection, Geophysics, 77, E203-E212, https://doi.org/10.1190/GEO20110247.1, 2012.

Oskooi, B. and Pedersen, L. B.: Comparison between VLF and RMT methods: A combined tool for mapping conductivity changes in the sedimentary cover, J. Appl. Geophys., 57, 227 241, https://doi.org/10.1016/j.jappgeo.2005.01.002, 2005.

Rasmussen, S., Nyboe, N. S., Mai, S., and Larsen, J. J.: Extraction and use of noise models from transient electromagnetic data, Geophysics, 83, E37-E46, https://doi.org/10.1190/GEO20170299.1, 2018a.

Rasmussen, S., Nyboe, N. S., Mai, S., and Larsen, J. J.: Robust cancellation of minimum shift keying-encoded radio interference in data from the transient electromagnetic method, Geophysics, 83, E87-E94, https://doi.org/10.1190/GEO2017-0611.1, 2018b. 